ANNALES

POLONICI MATHEMATICI

$81.1(2003)$

\title{
Loewner chains and quasiconformal extension of holomorphic mappings
}

\author{
by HidetakA Hamada (Kitakyusu) and \\ Gabriela KoHr (Cluj-Napoca)
}

Abstract. Let $f(z, t)$ be a Loewner chain on the Euclidean unit ball $B$ in $\mathbb{C}^{n}$. Assume that $f(z)=f(z, 0)$ is quasiconformal. We give a sufficient condition for $f$ to extend to a quasiconformal homeomorphism of $\mathbb{R}^{2 n}$ onto itself.

1. Introduction. Becker $[\mathrm{Be}]$ showed that if a holomorphic function $f$ on the unit disc $U$ satisfies

$$
\left|\frac{z f^{\prime \prime}(z)}{f^{\prime}(z)}\right| \leq \frac{c}{1-|z|^{2}} \quad(0<c<1),
$$

then $f$ is univalent on $U$ and extends to a quasiconformal homeomorphism of $\mathbb{C}$ onto itself. Pfaltzgraff [Pf2] generalized the above result to quasiregular locally biholomorphic mappings on the Euclidean unit ball $B$ in $\mathbb{C}^{n}$. He showed that if a quasiregular locally biholomorphic mapping $f$ on $B$ satisfies

$$
\left\|[D f(z)]^{-1} D^{2} f(z)(z, \cdot)\right\| \leq \frac{c}{1-\|z\|^{2}} \quad(0<c<1),
$$

then $f$ is biholomorphic on $B$ and extends to a quasiconformal homeomorphism of $\mathbb{R}^{2 n}$ onto itself. Chuaqui [Ch] obtained a quasiconformal extension of a quasiconformal strongly starlike mapping $f$ with $\left\|[D f(z)]^{-1} f(z)\right\|$ uniformly bounded on the Euclidean unit ball $B$ in $\mathbb{C}^{n}$. The first author [Ha] extended this result to a bounded balanced domain $\Omega$ in $\mathbb{C}^{n}$ with $C^{1}$ plurisubharmonic defining functions, and the authors [Ha-Ko3] generalized it to the unit ball with respect to an arbitrary norm on $\mathbb{C}^{n}$. The authors [Ha-Ko2] also gave a quasiconformal extension of a quasiconformal strongly spirallike mapping $f$ of type $\alpha$ with $\left\|[D f(z)]^{-1} f(z)\right\|$ uniformly bounded on a bounded balanced domain $\Omega$ in $\mathbb{C}^{n}$ with $C^{1}$ plurisubharmonic defining functions. To prove the above results, they imbed $f$ in a Loewner chain.

2000 Mathematics Subject Classification: 32H02, 30C65.

Key words and phrases: Loewner chain, quasiconformal extension. 
On the other hand, Graham-Hamada-Kohr [Gr-Ha-Ko] investigated a family of normalized biholomorphic mappings on the unit ball with respect to an arbitrary norm on $\mathbb{C}^{n}$ which arises in the study of Loewner chains, namely the family of mappings which have a parametric representation. For the case of the unit polydisc, these maps were studied by Poreda [Por1], [Por2]; on the Euclidean ball, they were studied extensively by Kohr [Ko]. In [Gr-Ha-Ko], growth and covering theorems for these mappings as well as coefficient estimates were obtained.

In this paper, we will give a sufficient condition for a normalized quasiconformal biholomorphic mapping $f$ on $B$ which can be imbedded in a Loewner chain to extend to a quasiconformal homeomorphism of $\mathbb{R}^{2 n}$ onto itself. We also show that the results in [Ch], [Ha-Ko2], [Ha-Ko3], [Pf2] and [Re-Ma] can be reduced to ours.

2. Preliminaries. Let $\mathbb{C}^{n}$ denote the space of $n$ complex variables $z=\left(z_{1}, \ldots, z_{n}\right)^{\prime}$ with the Euclidean inner product $\langle\cdot, \cdot\rangle$ and the Euclidean norm $\|z\|=\sqrt{\langle z, z\rangle}$. The symbol ' means the transpose of vectors and matrices. The origin $(0, \ldots, 0)^{\prime}$ is denoted by 0 , and $L\left(\mathbb{C}^{n}, \mathbb{C}^{m}\right)$ is the space of continuous linear operators from $\mathbb{C}^{n}$ into $\mathbb{C}^{m}$ with the standard operator norm. Let $I$ denote the identity in $L\left(\mathbb{C}^{n}, \mathbb{C}^{n}\right)$.

Let $B_{r}=\left\{z \in \mathbb{C}^{n}:\|z\|<r\right\}$ and let $B=B_{1}$. In the case of one complex variable, $B_{r}$ is denoted by $U_{r}$ and $U_{1}$ by $U$. If $G \subset \mathbb{C}^{n}$ is an open set, let $H(G)$ denote the set of holomorphic mappings from $G$ into $\mathbb{C}^{n}$. If $f \in H\left(B_{r}\right)$, we say that $f$ is normalized if $f(0)=0$ and $D f(0)=I$.

We recall that a mapping $F: B \times[0, \infty) \rightarrow \mathbb{C}^{n}$ is called a Loewner chain if $F(\cdot, t)$ is biholomorphic on $B, F(0, t)=0, D F(0, t)=e^{t} I$ for $t \geq 0$ and

$$
F(z, s) \prec F(z, t), \quad z \in B, 0 \leq s \leq t<\infty,
$$

where the symbol $\prec$ means the usual subordination.

It is known that starlikeness can be characterized in terms of Loewner chains: $f$ is a normalized starlike mapping if and only if $f(z, t)=e^{t} f(z)$, $z \in B, t \geq 0$, is a Loewner chain [Pf-Su]. For the analytical characterization of starlikeness, the reader may consult [Su1] and [Su2] (cf. [Ha]).

Now, we recall the notion of spirallikeness due to Gurganus [Gu] and Suffridge [Su3]. Let $A \in L\left(\mathbb{C}^{n}, \mathbb{C}^{n}\right)$ be such that $m(A)>0$, where

$$
m(A)=\inf \left\{\Re\langle A z, z\rangle: z \in \mathbb{C}^{n},\|z\|=1\right\} .
$$

A normalized locally biholomorphic mapping $f \in H(B)$ is called spirallike relative to $A$ if $f$ is biholomorphic on $B$ and $f(B)$ is a spirallike domain with respect to $A$, that is,

$$
e^{-s A} f(B) \subset f(B), \quad s \geq 0,
$$


where

$$
e^{-s A}=\sum_{k=0}^{\infty} \frac{(-1)^{k}}{k !} s^{k} A^{k} .
$$

Suffridge [Su3] showed that if $f$ is a normalized locally biholomorphic mapping on $B$, then $f$ is spirallike relative to a linear operator $A$ with $m(A)>0$ if and only if

$$
\Re\left\langle[D f(z)]^{-1} A f(z), z\right\rangle>0, \quad z \in B \backslash\{0\} .
$$

If $A=e^{-i \alpha} I$, where $\alpha \in \mathbb{R},|\alpha|<\pi / 2$, and $f$ is spirallike relative to $A$, we say that $f$ is spirallike of type $\alpha$ ([Ha-Ko1]).

The authors [Ha-Ko1] showed that spirallikeness of type $\alpha$ has the following characterization in terms of Loewner chains: $f$ is spirallike of type $\alpha$ if and only if

$$
f(z, t)=e^{(1-i a) t} f\left(e^{i a t} z\right), \quad z \in B, t \geq 0,
$$

is a Loewner chain, where $a=\tan \alpha$.

Let

$$
h(z, t)=i a z+(1-i a) e^{-i a t}\left[D f\left(e^{i a t} z\right)\right]^{-1} f\left(e^{i a t} z\right) .
$$

Then we have

$$
\Re\langle h(z, t), z\rangle=\frac{1}{\cos \alpha} \Re\left\{\left\langle e^{-i \alpha}\left[D f\left(e^{i a t} z\right)\right]^{-1} f\left(e^{i a t} z\right), e^{i a t} z\right\rangle\right\}>0
$$

for any $z \in B \backslash\{0\}$. Let $z \in \partial B$ and let $\zeta \in U \backslash\{0\}$. Then

$$
\Re\left\langle\frac{h(\zeta z, t)}{\zeta}, z\right\rangle=\frac{1}{|\zeta|^{2}} \Re(\langle h(\zeta z, t), \zeta z\rangle)>0 .
$$

Let

$$
\phi_{z}(\zeta, t)=\left\langle\frac{h(\zeta z, t)}{\zeta}, z\right\rangle
$$

for $\zeta \neq 0$ and $\phi_{z}(0, t)=1$. Since $h(0, t)=0$ and $D h(0, t)=I, \phi_{z}(\cdot, t)$ is a holomorphic function on $U$ and $\Re \phi_{z}(\zeta, t)>0$ for $\zeta \in U$ from (2.1).

If we put

$$
\sigma_{z}(\zeta, t)=\frac{\phi_{z}(\zeta, t)-1}{\phi_{z}(\zeta, t)+1},
$$

then $\sigma_{z}(\cdot, t)$ is a holomorphic function on $U$ such that $\sigma_{z}(0, t)=0$ and $\left|\sigma_{z}(\zeta, t)\right|<1$ for $\zeta \in U$.

DEFINITION 2.1. $f$ is said to be strongly spirallike of type $\alpha$ if $\phi_{z}(U, 0)$ is contained in a compact subset of the right half-plane independent of $z \in \partial B$. Or, equivalently, there exists a $c$ with $0<c<1$ such that $\left|\sigma_{z}(\zeta, 0)\right| \leq c$ uniformly for $z \in \partial B, \zeta \in U$.

When $\alpha=0$, the above definition coincides with the definition of strongly starlike mappings due to Chuaqui [Ch] (cf. [Ha]). 
Let $\mathcal{M}$ denote the well known set of mappings with "positive real part on $B "$, that is,

$$
\mathcal{M}=\{p \in H(B): p(0)=0, D p(0)=I, \Re\langle p(z), z\rangle>0, z \in B \backslash\{0\}\} .
$$

The following lemma is proved in Graham-Hamada-Kohr [Gr-Ha-Ko].

Lemma 2.2. For each $r \in(0,1)$ there exists a constant $M=M(r)$, independent of $p$, such that $\|p(z)\| \leq M(r)$ for $\|z\| \leq r, p \in \mathcal{M}$.

Using Lemma 2.2 and [Por3, Theorem 6], the authors of [Gr-Ha-Ko] obtained the following lemma.

Lemma 2.3. Let $h_{t}(z)=h(z, t): B \times[0, \infty) \rightarrow \mathbb{C}^{n}$ satisfy the following conditions:

(i) for each $t \geq 0, h_{t}(\cdot) \in \mathcal{M}$;

(ii) for each $z \in B, h(z, t)$ is a measurable function of $t \in[0, \infty)$.

Let $f_{t}(z)=f(z, t): B \times[0, \infty) \rightarrow \mathbb{C}^{n}$ be such that $f(\cdot, t) \in H(B), f(0, t)=0$, $D f(0, t)=e^{t} I$ for each $t \geq 0$ and $f(z, \cdot)$ is a locally Lipschitz continuous function of $t \in[0, \infty)$ locally uniformly with respect to $z \in B$. Suppose that

$$
\frac{\partial f}{\partial t}(z, t)=D f(z, t) h(z, t) \quad \text { a.e. } t \geq 0,
$$

for all $z \in B$. Further, assume that there exists an increasing sequence $\left\{t_{m}\right\}$ such that $t_{m}>0, t_{m} \rightarrow \infty$ and

$$
\lim _{m \rightarrow \infty} e^{-t_{m}} f\left(z, t_{m}\right)=F(z)
$$

locally uniformly on $B$. Then $f(z, t)$ is a Loewner chain and

$$
\lim _{t \rightarrow \infty} e^{t} v(z, s, t)=f(z, s)
$$

locally uniformly on $B$ for each $s \geq 0$, where $v=v(z, s, t)$ is the solution of the initial value problem

$$
\frac{\partial v}{\partial t}=-h(v, t) \quad \text { a.e. } t \geq s, \quad v(s)=z .
$$

REMARK 2.4. (i) In the case of one complex variable, every Loewner chain satisfies the assumptions of Lemma 2.3 (cf. [Pom, Theorem 6.2]).

(ii) Let $f(z, t)$ be a Loewner chain which is locally Lipschitz continuous in $t$ locally uniformly with respect to $z \in B$. In [Gr-Ha-Ko, Theorem 1.10], it was shown that there exists a mapping $h=h(z, t)$ such that $h(\cdot, t) \in \mathcal{M}$ for each $t \geq 0, h(z, t)$ is measurable in $t$ for each $z \in B$, and for almost all $t \geq 0$

$$
\frac{\partial f}{\partial t}(z, t)=D f(z, t) h(z, t) \quad \text { for all } z \in B .
$$

Therefore, if $\left\{e^{-t} f(z, t)\right\}_{t \geq 0}$ is a normal family, then $f(z, t)$ satisfies the assumptions of Lemma 2.3. 
Let $\Omega, \Omega^{\prime}$ be domains in $\mathbb{R}^{m}$. Let $\|\cdot\|$ be the Euclidean norm on $\mathbb{R}^{m}$ and $K>0$ be a constant. A homeomorphism $f: \Omega \rightarrow \Omega^{\prime}$ is said to be $K$-quasiconformal if it is differentiable a.e., ACL (absolutely continuous on lines) and

$$
\|D(f ; x)\|^{m} \leq K|\operatorname{det} D(f ; x)| \quad \text { a.e. in } \Omega,
$$

where $D(f ; x)$ denotes the (real) Jacobian matrix of $f$ and

$$
\|D(f ; x)\|=\sup \{\|D(f ; x)(a)\|:\|a\|=1\} .
$$

Let $G$ be a domain in $\mathbb{C}^{n}$ and let $K>0$ be a constant. A holomorphic mapping $f: G \rightarrow \mathbb{C}^{n}$ is said to be $K$-quasiregular if

$$
\|D f(z)\|^{n} \leq K|\operatorname{det} D f(z)|, \quad z \in G,
$$

where

$$
\|D f(z)\|=\sup \{\|D f(z)(a)\|:\|a\|=1\} .
$$

We remark that a $K$-quasiregular biholomorphic mapping is $K^{2}$-quasiconformal.

\section{Main results}

Lemma 3.1. Let $f(z, t)$ be a Loewner chain which satisfies the assumptions of Lemma 2.3. If there exists a constant $c_{1}>0$ such that

$$
c_{1}\|z\|^{2} \leq \Re\langle h(z, t), z\rangle
$$

for $z \in B \backslash\{0\}, t \geq 0$, then there exists a constant $d$ such that

$$
\|f(z, t)\| \leq d e^{t}\|z\|
$$

for $z \in B, t \geq 0$.

Proof. Since $h(\cdot, t) \in \mathcal{M}$,

$$
\|z\|^{2} \frac{1-\|z\|}{1+\|z\|} \leq \Re\langle h(z, t), z\rangle \leq\|z\|^{2} \frac{1+\|z\|}{1-\|z\|}
$$

for $z \in B \backslash\{0\}$ by [Pf1, Lemma 2.1]. Fix $s \geq 0$ and $z \in B \backslash\{0\}$ and let $v(t)=v(z, s, t)$ be the solution of the initial value problem (2.2). Then

$$
\frac{\partial\|v\|}{\partial t}=-\frac{1}{\|v\|} \Re\langle h(v, t), v\rangle<0 \quad \text { a.e. on }[s, \infty) .
$$

Since $\|v(t)\| \rightarrow 0$ as $t \rightarrow \infty$, there exists a $t_{0}>0$ such that $\|v(t)\|<1 / 2$ for $t \geq t_{0}$. If $\|z\|>1 / 2$, then, for $t>t_{0}$, we have 


$$
\begin{aligned}
\log \left(\frac{\left\|e^{t-s} v(z, s, t)\right\|}{\|z\|}\right) & =-\int_{s}^{t} \frac{\|v(\tau)\|}{\Re\langle h(v(\tau)), v(\tau)\rangle} \frac{d\|v(\tau)\|}{d \tau} d \tau-\int_{\|v(t)\|}^{\|z\|} \frac{1}{x} d x \\
& \leq \int_{\|v(t)\|}^{1 / 2} \frac{1+x}{x(1-x)} d x+\int_{1 / 2}^{\|z\|} \frac{1}{c_{1} x} d x-\int_{\|v(t)\|} \frac{1}{x} d x \\
& \leq \int_{0}^{1 / 2} \frac{2}{1-x} d x+\int_{1 / 2}^{1} \frac{1}{c_{1} x} d x \leq\left(2+\frac{1}{c_{1}}\right) \log 2
\end{aligned}
$$

by the assumption and (3.1). If $\|z\| \leq 1 / 2$, then, for $t>t_{0}$, we have

$$
\begin{aligned}
\log \left(\frac{\left\|e^{t-s} v(z, s, t)\right\|}{\|z\|}\right) & =-\int_{s}^{t} \frac{\|v(\tau)\|}{\Re\langle h(v(\tau)), v(\tau)\rangle} \frac{d\|v(\tau)\|}{d \tau} d \tau-\int_{\|v(t)\|}^{\|z\|} \frac{1}{x} d x \\
& \leq \int_{\|v(t)\|}^{\|z\|} \frac{1+x}{x(1-x)} d x-\int_{\|v(t)\|}^{\|z\|} \frac{1}{x} d x \\
& \leq \int_{0}^{1 / 2} \frac{2}{1-x} d x \leq 2 \log 2
\end{aligned}
$$

by (3.1). Letting $t \rightarrow \infty$, we obtain the conclusion.

Let $f(z, t)$ be a Loewner chain such that $f(z, 0)$ is quasiconformal. The following theorem gives a sufficient condition for $f(z, 0)$ to have a quasiconformal extension to $\mathbb{R}^{2 n}$.

THEOREM 3.2. Let $f(z, t)$ be a Loewner chain which satisfies the assumptions of Lemma 2.3. Assume that the following conditions are satisfied:

$$
\|D f(z, t)\| \leq \frac{M_{1}(t)}{(1-\|z\|)^{\alpha}}, \quad z \in B, t \geq 0,
$$

where $M_{1}(t)$ is locally bounded with respect to $t$ and $\alpha$ is a constant with $0 \leq \alpha<1$

(ii) there exists a constant $c_{1}>0$ such that

$$
c_{1}\|z\|^{2} \leq \Re\langle h(z, t), z\rangle
$$

for $z \in B \backslash\{0\}, t \geq 0$;

(iii) there exists a constant $c_{2}>0$ such that $\|h(z, t)\| \leq c_{2}$ for $z \in B$, $t \geq 0$

(iv) $f(z, t)$ is $K_{1}$-quasiconformal for each $t$.

Then $f(z, t)$ has a continuous extension to $\bar{B}$ (again denoted by $f(z, t)$ ) 
and

$$
F(z)= \begin{cases}f(z, 0), & z \in \bar{B} \\ f(z /\|z\|, \log \|z\|), & z \notin \bar{B}\end{cases}
$$

is a quasiconformal homeomorphism of $\mathbb{R}^{2 n}$ onto itself.

Proof. By assumption (i) and [Pf2, Lemma 2.2], $f_{t}(z)=f(z, t)$ has a continuous extension to $\bar{B}$ and

$$
\|f(z, t)-f(w, t)\| \leq M_{2}(t)\|z-w\|^{1-\alpha}
$$

for $z, w \in \bar{B}$, where $M_{2}(t)$ is locally bounded on $[0, \infty)$.

Fix $s \geq 0$ and $z \in B \backslash\{0\}$ and let $v(t)=v(z, s, t)$ be the solution of the initial value problem (2.2). Then

$$
\frac{\partial\|v\|}{\partial t}=-\frac{1}{\|v\|} \Re\langle h(v, t), v\rangle \leq-c_{1}\|v\| \quad \text { a.e. on }[s, \infty)
$$

by assumption (ii). Then we have

$$
\|v(z, s, t)\| \leq\|z\| e^{-c_{1}(t-s)}
$$

for $0 \leq s<t$. Therefore, $\overline{v(B, s, t)} \subset B$ for $0 \leq s<t$. Since $f_{s}(z)=$ $f_{t}(v(z, s, t))$ for $0 \leq s<t, z \in B$, and $f_{s}$ is continuous on $\bar{B}$, we have $f_{s}(\bar{B}) \subset f_{t}(B)$ for $0 \leq s<t$. Then

$$
v(z, s, t)=f_{t}^{-1}\left(f_{s}(z)\right), \quad z \in \bar{B}
$$

defines a continuous extension of $v$ to $\bar{B}$. For $z \in B$, we have

$$
\|z-v(z, s, t)\|=\left\|\int_{s}^{t} h(v, \tau) d \tau\right\| \leq c_{2}|t-s|
$$

for $0 \leq s<t$ by assumption (iii). Since $v$ is continuous on $\bar{B}$, this estimate holds for $z \in \bar{B}$. Suppose that $f_{s}\left(z_{1}\right)=f_{s}\left(z_{2}\right)$ for $z_{1}, z_{2} \in \bar{B}$. Then for $t>s$, we have

$$
f_{t}\left(v\left(z_{1}, s, t\right)\right)=f_{t}\left(v\left(z_{2}, s, t\right)\right) .
$$

Since $v(\bar{B}, s, t) \subset B$ for $0 \leq s<t$ and $f_{t}$ is univalent on $B$, we obtain $v\left(z_{1}, s, t\right)=v\left(z_{2}, s, t\right)$. Letting $t \rightarrow s$, we have $z_{1}=z_{2}$ by (3.4). Therefore, $f_{s}$ is univalent on $\bar{B}$.

For $1 / 2<r<1$, let $f^{r}(z, t)=r^{-1} f(r z, t)(t \geq 0)$ and let

$$
F^{r}(z)= \begin{cases}f^{r}(z, 0), & z \in \bar{B}, \\ f^{r}(z /\|z\|, \log \|z\|), & z \notin \bar{B} .\end{cases}
$$

We will show that $F^{r}$ converges uniformly on compact subsets of $\mathbb{R}^{2 n}$ to $F$ as $r \rightarrow 1$, is ACL, is differentiable a.e., has outer dilatation bounded a.e. with a bound independent of $r$, and is a homeomorphism of $\mathbb{R}^{2 n}$ onto itself. Then by [Vä, Theorem 21.7 and Corollary 37.4], $F$ is a quasiconformal homeomorphism of $\mathbb{R}^{2 n}$ onto itself, since $F \mid B=f(z, 0)$ is nonconstant. 
By Lemma 3.1 and assumption (ii), we have

$$
\|f(z, s)\| \leq c_{3} e^{s}\|z\|
$$

on $\bar{B} \times[0, \infty)$ for some constant $c_{3}$. Let $z \in \bar{B}$. Then

$$
\begin{aligned}
\left\|F^{r}(z)-F(z)\right\| & =\left\|r^{-1} f(r z, 0)-f(z, 0)\right\| \\
& \leq r^{-1}\|f(r z, 0)-f(z, 0)\|+\left(r^{-1}-1\right)\|f(z, 0)\| \\
& \leq r^{-1} M_{2}(0)\|r z-z\|^{1-\alpha}+\left(r^{-1}-1\right) c_{3}\|z\|
\end{aligned}
$$

by (3.2) and (3.5). Let $z \in \mathbb{R}^{2 n} \backslash B$. Then

$$
\begin{aligned}
\left\|F^{r}(z)-F(z)\right\|= & \left\|r^{-1} f\left(r \frac{z}{\|z\|}, \log \|z\|\right)-f\left(\frac{z}{\|z\|}, \log \|z\|\right)\right\| \\
\leq & r^{-1}\left\|f\left(r \frac{z}{\|z\|}, \log \|z\|\right)-f\left(\frac{z}{\|z\|}, \log \|z\|\right)\right\| \\
& +\left(r^{-1}-1\right)\left\|f\left(\frac{z}{\|z\|}, \log \|z\|\right)\right\| \\
\leq & r^{-1} M_{2}(\log \|z\|)(1-r)^{1-\alpha}+\left(r^{-1}-1\right) c_{3}\|z\|
\end{aligned}
$$

by (3.2) and (3.5). Thus, $F^{r}$ converges uniformly on compact subsets of $\mathbb{R}^{2 n}$ to $F$.

Since $\left\|D f^{r}(z, t)\right\|=\|D f(r z, t)\| \leq M_{1}(t) /(1-r)^{\alpha}$ by assumption $(\mathrm{i})$, we have

$$
\left\|f^{r}(z, t)-f^{r}(w, t)\right\| \leq \frac{M_{3}(t)}{(1-r)^{\alpha}}\|z-w\|
$$

for $z, w \in \bar{B}$, where $M_{3}(t)$ is locally bounded on $[0, \infty)$. Using the relation $f(z, s)=f(v(z, s, t), t)$ for $0 \leq s<t,(3.4)$ and (3.6), we have

$$
\begin{aligned}
\left\|f^{r}(z, t)-f^{r}(z, s)\right\| & =r^{-1}\|f(r z, t)-f(r z, s)\| \\
& =r^{-1}\|f(r z, t)-f(v(r z, s, t), t)\| \\
& =\left\|f^{r}(z, t)-f^{r}\left(r^{-1} v(r z, s, t), t\right)\right\| \\
& \leq \frac{M_{3}(t)}{(1-r)^{\alpha}}\left\|z-r^{-1} v(r z, s, t)\right\| \\
& \leq \frac{c_{2} M_{3}(t)}{r(1-r)^{\alpha}}|t-s|
\end{aligned}
$$

for $z \in \bar{B}, 0 \leq s<t$. If $z, w \in \bar{B}$, then

$$
\left\|F^{r}(z)-F^{r}(w)\right\|=\left\|f^{r}(z, 0)-f^{r}(w, 0)\right\| \leq \frac{M_{3}(0)}{(1-r)^{\alpha}}\|z-w\|
$$

by (3.6). If $z, w \in \mathbb{R}^{2 n} \backslash B$ and $\|z\| \geq\|w\|$, then 


$$
\begin{aligned}
\left\|F^{r}(z)-F^{r}(w)\right\|= & \left\|f^{r}\left(\frac{z}{\|z\|}, \log \|z\|\right)-f^{r}\left(\frac{w}{\|w\|}, \log \|w\|\right)\right\| \\
\leq & \left\|f^{r}\left(\frac{z}{\|z\|}, \log \|z\|\right)-f^{r}\left(\frac{w}{\|w\|}, \log \|z\|\right)\right\| \\
& +\left\|f^{r}\left(\frac{w}{\|w\|}, \log \|z\|\right)-f^{r}\left(\frac{w}{\|w\|}, \log \|w\|\right)\right\| \\
\leq & \left(2+r^{-1} c_{2}\right) \frac{M_{3}(\log \|z\|)}{(1-r)^{\alpha}}\|z-w\|
\end{aligned}
$$

by (3.6) and (3.7). If $z \in B, w \in \mathbb{R}^{2 n} \backslash \bar{B}$, then there exists a real number $t$ with $0<t<1$ such that $\zeta=(1-t) z+t w \in \partial B$. Therefore,

$$
\begin{aligned}
\left\|F^{r}(z)-F^{r}(w)\right\| & \leq\left\|F^{r}(z)-F^{r}(\zeta)\right\|+\left\|F^{r}(\zeta)-F^{r}(w)\right\| \\
& \leq \frac{M_{3}(0)}{(1-r)^{\alpha}}\|z-\zeta\|+\left(2+r^{-1} c_{2}\right) \frac{M_{3}(\log \|w\|)}{(1-r)^{\alpha}}\|\zeta-w\| \\
& \leq \frac{1}{(1-r)^{\alpha}}\left\{M_{3}(0)+\left(2+r^{-1} c_{2}\right) M_{3}(\log \|w\|)\right\}\|z-w\|
\end{aligned}
$$

by (3.8) and (3.9). Thus, $F^{r}$ satisfies a local Lipschitz condition (with exponent 1 ) in $\mathbb{R}^{2 n}$. Hence $F^{r}$ is ACL in $\mathbb{R}^{2 n}$ and by a theorem of RademacherStepanov [Sa, p. 311], it is (real) differentiable a.e. in $\mathbb{R}^{2 n}$.

Next, we will show that $F^{r}$ has outer dilatation bounded a.e. with a bound independent of $r$. Let $f_{t}=u_{t}+i v_{t}$ and let $F^{r}=u^{r}+i v^{r}$. Then we have

$$
\left\|D\left(u^{r}, v^{r} ; x, y\right)\right\|^{2 n} \leq K_{1}\left|\operatorname{det} D\left(u^{r}, v^{r} ; x, y\right)\right| \quad \text { in } \bar{B},
$$

since

$$
D\left(u^{r}, v^{r} ; x, y\right)=D\left(u_{0}, v_{0} ; r x, r y\right) \quad \text { in } \bar{B}
$$

and $f_{t}$ is $K_{1}$-quasiconformal in $B$. For $z \notin \bar{B}$, let $\zeta=r\|z\|^{-1} z \in B \backslash\{0\}$ and let $\zeta=\xi+i \eta$. Then

$$
D\left(u^{r}, v^{r} ; x, y\right)=\|z\|^{-1} D\left(u_{t}, v_{t} ; \xi, \eta\right)(I+M(\xi, \eta)),
$$

where $t=\log \|z\|$ and

$$
M(\xi, \eta)=r^{-1}\left(\begin{array}{c}
\Re(h(\zeta, t)-\zeta) \\
\Im(h(\zeta, t)-\zeta)
\end{array}\right) \operatorname{grad}\|(x, y)\|,
$$

as $\partial f(z, t) / \partial t=D f(z, t) h(z, t)$. Since $M(\xi, \eta)$ has rank 1 ,

$$
\begin{aligned}
\operatorname{det}(I+M(\xi, \eta)) & =1+\operatorname{tr} M(\xi, \eta)=r^{-1}\|\zeta\|^{-1} \Re\langle h(\zeta, t), \zeta\rangle \\
& \geq c_{1} r^{-1}\|\zeta\| \geq c_{1}
\end{aligned}
$$

by Lemma 3.1 and assumption (ii). Then we have

$$
\begin{aligned}
\left|\operatorname{det} D\left(u^{r}, v^{r} ; x, y\right)\right| & =\|z\|^{-2 n}\left|\operatorname{det} D\left(u_{t}, v_{t} ; \xi, \eta\right)\right||\operatorname{det}(I+M(\xi, \eta))| \\
& \geq\|z\|^{-2 n} c_{1}\left|\operatorname{det} D\left(u_{t}, v_{t} ; \xi, \eta\right)\right| .
\end{aligned}
$$


Since $\operatorname{grad}\|(x, y)\|$ is uniformly bounded, $\|M(\xi, \eta)\|=r^{-1}\|h(\zeta, t)-\zeta\|$. $\|\operatorname{grad}\|(x, y)\|\|$ is uniformly bounded for $r$ near 1 by assumption (iii). Thus

$$
\begin{aligned}
\left\|D\left(u^{r}, v^{r} ; x, y\right)\right\| & \leq\|z\|^{-1}\left\|D\left(u_{t}, v_{t} ; \xi, \eta\right)\right\|\|I+M(\xi, \eta)\| \\
& \leq K_{2}\|z\|^{-1}\left\|D\left(u_{t}, v_{t} ; \xi, \eta\right)\right\|
\end{aligned}
$$

for some constant $K_{2}>0$.

By (3.10), (3.11) and assumption (iv), we have

$$
\begin{aligned}
\left\|D\left(u^{r}, v^{r} ; x, y\right)\right\|^{2 n} & \leq K_{2}^{2 n}\|z\|^{-2 n}\left\|D\left(u_{t}, v_{t} ; \xi, \eta\right)\right\|^{2 n} \\
& \leq K_{1} K_{2}^{2 n}\|z\|^{-2 n}\left|\operatorname{det} D\left(u_{t}, v_{t} ; \xi, \eta\right)\right| \\
& \leq K_{1} K_{2}^{2 n} c_{1}^{-1}\left|\operatorname{det} D\left(u^{r}, v^{r} ; x, y\right)\right|
\end{aligned}
$$

on $\mathbb{R}^{2 n} \backslash \bar{B}$.

Finally, we will show that $F^{r}$ is a homeomorphism of $\mathbb{R}^{2 n}$ onto itself. It is clear that $F^{r}$ is continuous on $\mathbb{R}^{2 n}$. From (3.3), we have

$$
f_{s}\left(\bar{B}_{r}\right) \subset f_{t}\left(B_{r}\right) \quad \text { for } 0<r<1 \text { and } 0 \leq s<t .
$$

Using (3.12) and the fact that $f_{t}$ is univalent on $B$, we can show that $F^{r}$ is univalent on $\mathbb{R}^{2 n}$. By [Pf1, Lemma 2.2], we have

$$
\|f(z, s)\|=\lim _{t \rightarrow \infty} e^{t}\|v(z, s, t)\| \geq e^{s} \frac{\|z\|}{(1+\|z\|)^{2}}
$$

for all $z \in B, s \geq 0$. Then

$$
\left\|F^{r}(z)\right\|=r^{-1}\left\|f\left(r \frac{z}{\|z\|}, \log \|z\|\right)\right\| \geq \frac{R}{(1+r)^{2}}
$$

on $\|z\|=R$ with $R \geq 1$. Since

$$
F^{r}(\{\|z\|=R\})=r^{-1} f_{\log R}(\{\|z\|=r\})
$$

for any $R>1, F^{r}(\{\|z\|=R\})$ is the boundary of the domain $G_{R}=$ $r^{-1} f_{\log R}(\{\|z\|<r\})$. By (3.12), we have $F^{r}(\{1<\|z\|<R\}) \subset G_{R} \backslash F^{r}(\bar{B})$. Hence $F^{r}(\{1<\|z\|<R\})=G_{R} \backslash F^{r}(\bar{B})$, since $F^{r}$ is a local diffeomorphism on $\mathbb{R}^{2 n} \backslash \bar{B}$ by (3.10), continuous and univalent on $\mathbb{R}^{2 n}$. Then using (3.13), we can show that $F^{r}$ is a surjective map onto $\mathbb{R}^{2 n}$. Let $\mathbb{R}^{2 n} \cup\{\infty\}=S^{2 n}$ be a one-point compactification of $\mathbb{R}^{2 n}$. We extend $F^{r}$ to $S^{2 n}$ by $F^{r}(\infty)=\infty$. Then $F^{r}$ is a continuous bijective mapping from $S^{2 n}$ onto itself by (3.13). Therefore, $F^{r}$ is a homeomorphism from $S^{2 n}$ onto itself. Thus $F^{r}$ is a homeomorphism from $\mathbb{R}^{2 n}$ onto itself. This completes the proof.

REMARK 3.3. If there exists an $E(z, t): B \times[0, \infty) \rightarrow L\left(\mathbb{C}^{n}, \mathbb{C}^{n}\right)$ which is holomorphic in $z, E(0, t)=0$ for $t \geq 0$ and $\|E(z, t)\| \leq c<1$ for $z \in B$, $t \geq 0$ such that $h(z, t)=[I-E(z, t)]^{-1}[I+E(z, t)](z)$ for $z \in B, t \geq 0$, then we can show that conditions (ii) and (iii) of Theorem 3.2 are satisfied as in [Pf1]. 
EXAMPLE 3.4. We give an example which shows that condition (ii) in Theorem 3.2 cannot be omitted. Let $B$ be the Euclidean unit ball in $\mathbb{C}^{2}$. Let

$$
f(z)=\left(z_{1}+a z_{2}^{2}, z_{2}\right)^{\prime} .
$$

Then $f$ is starlike if and only if $|a| \leq 3 \sqrt{3} / 2$ by Example 3 in [Su3]. Put $a=3 \sqrt{3} / 2$. Since $f$ is starlike, $f(z, t)=e^{t} f(z)$ is a Loewner chain which satisfies the assumptions of Lemma 2.3. Since $D f(z, t)=e^{t} D f(z)$ and $f$ is a polynomial, condition (i) is satisfied. As $h(z, t)=[D f(z)]^{-1} f(z)=$ $\left(z_{1}-a z_{2}^{2}, z_{2}\right)^{\prime}$, condition (iii) is satisfied. Because $\|D f(z)\|$ is uniformly bounded in $B^{2}$, det $D f(z)=1$ and $D f(z, t)=e^{t} D f(z)$, condition (iv) is satisfied. Since $\langle h(z, t), z\rangle=\|z\|^{2}-a \bar{z}_{1} z_{2}^{2}$ tends to 0 as $\left(z_{1}, z_{2}\right) \rightarrow(1 / \sqrt{3}, \sqrt{2 / 3})$, condition (ii) is not satisfied. Further, we show that

$$
F(z)= \begin{cases}f(z, 0), & z \in \bar{B}, \\ f(z /\|z\|, \log \|z\|), & z \notin \bar{B},\end{cases}
$$

is not quasiconformal. For $z \notin \bar{B}, F(z)=\left(z_{1}+a z_{2}^{2} /\|z\|, z_{2}\right)$. By a direct computation, we have $\operatorname{det} D F(x, y)=0$ for $z_{1}=k / \sqrt{3}, z_{2}=\sqrt{2} k / \sqrt{3}$ with $k>1$, but $\|D F(x, y)\| \neq 0$. This implies that $F$ is not quasiconformal.

4. Applications. Ren-Ma [Re-Ma, Theorem 2] obtained the following theorem. We prove this result by using Theorem 3.2.

TheOREM 4.1. Let $f: B \rightarrow \mathbb{C}^{n}$ be a normalized holomorphic mapping on $B$ and let $G(z)$ be a nonsingular $n \times n$ matrix, holomorphic as a function of $z \in B$. Suppose that $G(0)=I$ and the following assumptions hold:

(i) $\left\|[G(z)]^{-1} D f(z)-I\right\| \leq c, z \in B$;

(ii) \|\|$z\left\|^{2}\left[[G(z)]^{-1} D f(z)-I\right]+\left(1-\|z\|^{2}\right)[G(z)]^{-1} D G(z)(z, \cdot)\right\| \leq c$, $z \in B$, where $0 \leq c<1$;

(iii) there exists a $K \geq 1$ such that $\|G(z)\|^{n} \leq K|\operatorname{det} G(z)|, z \in B$.

Then $f$ is univalent and quasiregular on $B$ and extends to a quasiconformal homeomorphism of $\mathbb{R}^{2 n}$ onto itself.

Proof. We will show that

$$
f(z, t)=f\left(z e^{-t}\right)+G\left(z e^{-t}\right)\left(e^{t}-e^{-t}\right) z
$$

satisfies the conditions of Theorem 3.2.

Indeed, $f(\cdot, t) \in H(B), f(0, t)=0, D f(0, t)=e^{t} I, t \geq 0$, and $f(z, \cdot) \in$ $C^{\infty}([0, \infty)), z \in B$. On the other hand, it is obvious that $\lim _{t \rightarrow \infty} e^{-t} f(z, t)$ $=z$ locally uniformly on $B$. Straightforward computations show that

$$
\frac{\partial f}{\partial t}(z, t)=D f(z, t) h(z, t), \quad z \in B, t \geq 0
$$


where $h(z, t)=[I-E(z, t)]^{-1}[I+E(z, t)](z)$, and

$$
\begin{aligned}
E(z, t)= & -e^{-2 t}\left\{\left[G\left(z e^{-t}\right)\right]^{-1} D f\left(z e^{-t}\right)-I\right\} \\
& -\left(1-e^{-2 t}\right)\left[G\left(z e^{-t}\right)\right]^{-1} D G\left(z e^{-t}\right)\left(z e^{-t}, \cdot\right)
\end{aligned}
$$

for all $z \in B$ and $t \geq 0$. Using condition (i), we see that

$$
\|E(z, 0)\| \leq c<1, \quad z \in B
$$

and using the maximum modulus theorem for holomorphic mappings into complex Banach spaces and condition (ii), we deduce for $t>0$ that

$$
\|E(z, t)\| \leq \max _{\|z\|=1}\|E(z, t)\| \leq c<1 .
$$

Thus $\|E(z, t)\| \leq c<1$ for $z \in B$ and $t \geq 0$. Since $E(0, t)=0$ for $t \geq 0$, conditions (ii) and (iii) of Theorem 3.2 are satisfied by Remark 3.3. Hence $f(z, t)$ is a Loewner chain by Lemma 2.3 .

Moreover, in view of the second condition in the hypothesis, we deduce that

$$
\left(1-\|z\|^{2}\right)\left\|[G(z)]^{-1} D G(z)(z, \cdot)\right\| \leq 2 c .
$$

Then with a similar reasoning to the one in [Pf2, Theorem 2.1], we conclude that there exists an absolute constant $M>0$ such that

$$
\|G(z)\| \leq \frac{M}{(1-\|z\|)^{c}}, \quad z \in B .
$$

Hence we obtain

$$
\begin{aligned}
\|D f(z, t)\| & =e^{t}\left\|G\left(z e^{-t}\right)(I-E(z, t))\right\| \\
& \leq e^{t} \frac{M}{\left(1-\left\|z e^{-t}\right\|\right)^{c}}(1+c) \leq \frac{M(1+c) e^{t}}{(1-\|z\|)^{c}},
\end{aligned}
$$

and therefore the first condition from Theorem 3.2 holds.

It remains to show that $f(z, t)$ is $K_{1}$-quasiconfomal in $B$ for all $t \geq 0$. Indeed, from the third condition in the hypothesis, it is easy to deduce that

$$
\begin{aligned}
\|D f(z, t)\|^{n} & =e^{n t}\left\|G\left(z e^{-t}\right)(I-E(z, t))\right\|^{n} \\
& \leq e^{n t}\left\|G\left(z e^{-t}\right)\right\|^{n}(1+c)^{n} \leq e^{n t} K\left|\operatorname{det} G\left(z e^{-t}\right)\right|(1+c)^{n} \\
& =\frac{|\operatorname{det} D f(z, t)|}{|\operatorname{det}[I-E(z, t)]|}(1+c)^{n} K \\
& \leq K\left(\frac{1+c}{1-c}\right)^{n}|\operatorname{det} D f(z, t)| .
\end{aligned}
$$

This completes the proof.

In particular, we obtain the following corollary.

COROLlary 4.2. Let $f: B \rightarrow \mathbb{C}^{n}$ be a normalized holomorphic mapping on $B$ and let $a: B \rightarrow \mathbb{C}$ be a holomorphic function such that $a(z) \neq 0$, $z \in B$, and $a(0)=1$. Suppose that the following assumptions hold: 
(i) $\|D f(z)-a(z) I\| \leq c|a(z)|, z \in B$;

(ii) \|\|$z\left\|^{2}[D f(z)-a(z) I]+\left(1-\|z\|^{2}\right) \frac{d a}{d z}(z) z^{\prime}\right\| \leq c|a(z)|$, where $0 \leq c<1$.

Then $f$ is univalent and quasiregular on $B$ and extends to a quasiconformal homeomorphism of $\mathbb{R}^{2 n}$ onto itself.

In particular, for $a(z) \equiv 1$, we obtain a result due to Brodskiu [Br].

If $G(z)=D f(z), z \in B$, in Theorem 4.1, we get Pfaltzgraff's quasiconformal extension result [Pf2, Theorem 2.1]. In this case, condition (iii) is equivalent to the condition that $f$ is quasiregular on $B$.

We next consider quasiconformal extension of quasiconformal strongly spirallike mappings of type $\alpha$. The authors [Ha-Ko2] obtained the following theorem. We prove this result by using Theorem 3.2.

THEOREM 4.3. Let $f$ be a quasiconformal, strongly spirallike mapping of type $\alpha$ with $\left\|[D f(z)]^{-1} f(z)\right\|$ uniformly bounded on $B$. Then $f(z)$ has a continuous extension to $\bar{B}$ (again denoted by $f$ ) and

$$
F(z)= \begin{cases}f(z), & z \in \bar{B}, \\ \|z\|^{1-i a} f\left(z /\|z\|^{1-i a}\right), & z \notin \bar{B}\end{cases}
$$

is a quasiconformal homeomorphism of $\mathbb{R}^{2 n}$ onto itself.

Proof. Let

$$
f(z, t)=e^{(1-i a) t} f\left(e^{i a t} z\right), \quad z \in B, t \geq 0,
$$

where $a=\tan \alpha$ and

$$
h(z, t)=i a z+(1-i a) e^{-i a t}\left[D f\left(e^{i a t} z\right)\right]^{-1} f\left(e^{i a t} z\right) .
$$

Then it is easy to show that $f(z, t)$ is a Loewner chain which satisfies the assumptions of Lemma 2.3. We will show that $f(z, t)$ satisfies the assumptions of Theorem 3.2. Clearly, $\|h(z, t)\|$ is uniformly bounded for $z \in B, t \geq 0$. This implies assumption (iii). Let $\phi_{z}$ and $\sigma_{z}$ be as in Section 2. There exists a constant $c$ such that $\left|\sigma_{z}(\zeta, 0)\right| \leq c<1$ uniformly for $z \in \partial B$. Since

$$
\begin{aligned}
\Re\langle h(z, t), z\rangle & =\Re\left\{\frac{1}{e^{i a t}}\left\langle h\left(e^{i a t} z, 0\right), z\right\rangle\right\} \\
& =\|z\|^{2} \Re \phi_{\widetilde{z}}(\|z\|, 0), \quad \widetilde{z}=e^{i a t} z /\|z\|,
\end{aligned}
$$

for $z \in B \backslash\{0\}$, we obtain

$$
\|z\|^{2} \frac{1-c\|z\|}{1+c\|z\|} \leq \Re\langle h(z, t), z\rangle \leq\|z\|^{2} \frac{1+c\|z\|}{1-c\|z\|}, \quad z \in B \backslash\{0\},
$$

by applying the Schwarz lemma to $\sigma_{\widetilde{z}}(\cdot, 0)$ as in Pfaltzgraff [Pf1, Lemma 2.1]. This implies assumption (ii). 
Let $w(z)=[D f(z)]^{-1} f(z)$. Since $h(z, 0)=i a z+(\cos \alpha)^{-1} e^{-i \alpha} w(z)$, we have

$$
\|z\|^{2} \frac{1-c\|z\|}{1+c\|z\|} \leq \frac{1}{\cos \alpha} \Re\left\langle e^{-i \alpha} w(z), z\right\rangle \leq\|z\|^{2} \frac{1+c\|z\|}{1-c\|z\|}
$$

for $z \in B \backslash\{0\}$ from (4.1). Therefore,

$$
\|z\| \frac{1-c\|z\|}{1+c\|z\|} \leq \frac{\|w(z)\|}{\cos \alpha} .
$$

Also, from (4.1) and Lemma 3.1, we have $\|f(z, t)\| \leq d e^{t}\|z\|$ for $z \in B$, $t \geq 0$, where $d$ is a constant. Then, using $D f(z) w(z)=f(z)$, we have

$$
\left\|D f(z)\left(\frac{w(z)}{\|w(z)\|}\right)\right\|=\frac{\|f(z)\|}{\|w(z)\|} \leq \frac{d(1+c)}{\cos \alpha(1-c)}
$$

for $z \neq 0$. By the Cauchy-Riemann equations, this implies that

$$
\left\|D(u, v ; x, y)\left(\Re \frac{w}{\|w\|}, \Im \frac{w}{\|w\|}\right)^{\prime}\right\| \leq \frac{d(1+c)}{\cos \alpha(1-c)}
$$

for $z \neq 0$, where $f=u+i v$ and $z=x+i y$. Since $D(u, v ; x, y)^{\prime} D(u, v ; x, y)$ is a positive semi-definite symmetric matrix, its eigenvalues are real and nonnegative. Denote them by $\lambda_{1}^{2}(x, y), \ldots, \lambda_{2 n}^{2}(x, y)$, where $0 \leq \lambda_{1}(x, y) \leq$ $\ldots \leq \lambda_{2 n}(x, y)$. Since $f$ is quasiconformal, we have $\lambda_{2 n}(x, y) \leq K \lambda_{1}(x, y)$ for some constant $K$. Then

$$
\|D(u, v ; x, y)\|=\lambda_{2 n}(x, y) \leq K \lambda_{1}(x, y) \leq K \frac{d(1+c)}{\cos \alpha(1-c)}
$$

for $z \neq 0$ from (4.2). Thus $\|D f(z)\|$ is uniformly bounded in $B$. Since $D f(z, t)=e^{t} D f\left(e^{i a t} z\right)$, we have $\|D f(z, t)\| \leq M e^{t}$ for $z \in B, t \geq 0$ and $f(z, t)$ is $K_{1}$-quasiconformal for $t \geq 0$, where $K_{1}$ is a positive constant. This implies assumptions (i) and (iv), and completes the proof.

If we put $\alpha=0$ in Theorem 4.3, we obtain the following corollary, which was obtained in [Ch] (cf. [Ha], [Ha-Ko3]).

COROLLARY 4.4. Let $f$ be a quasiconformal, strongly starlike mapping with $\|\left[D f(z)^{-1} f(z) \|\right.$ uniformly bounded on $B$. Then $f(z)$ has a continuous extension to $\bar{B}$ (again denoted by $f$ ) and

$$
F(z)= \begin{cases}f(z), & z \in \bar{B} \\ \|z\| f(z /\|z\|), & z \notin \bar{B}\end{cases}
$$

is a quasiconformal homeomorphism of $\mathbb{R}^{2 n}$ onto itself.

We remark that the mapping in Example 3.4 shows that the assumption of strong starlikeness in the above corollary cannot be omitted.

REMARK 4.5. We now consider generalizations of our results to the unit ball $B$ with respect to an arbitrary norm $\|\cdot\|$ on $\mathbb{C}^{n}$. For each $z \in \mathbb{C}^{n} \backslash\{0\}$, 
let

$$
T(z)=\left\{z^{*} \in L\left(\mathbb{C}^{n}, \mathbb{C}\right): z^{*}(z)=\|z\|,\left\|z^{*}\right\|=1\right\}
$$

This set is nonempty, by the well known Hahn-Banach theorem. Let

$$
\begin{aligned}
& \mathcal{M}=\left\{p \in H(B): p(0)=0, D p(0)=I, \Re z^{*}(p(z))>0,\right. \\
&\left.z \in B \backslash\{0\}, z^{*} \in T(z)\right\} .
\end{aligned}
$$

Then Lemmas 2.2 and 2.3 hold without any change. If we replace the condition

$$
c_{1}\|z\|^{2} \leq \Re\langle h(z, t), z\rangle
$$

by

$$
c_{1}\|z\| \leq \Re z^{*}(h(z, t)),
$$

then Lemma 3.1 and Theorem 3.2 hold. Remark 3.3, Theorem 4.1 and Corollary 4.2 also hold if $c<1 / 3$. Theorem 4.3 and Corollary 4.4 hold without any change.

Acknowledgements. The first author is partially supported by Grantin-Aid for Scientific Research (C) no. 14540195 from the Japan Society for the Promotion of Science, 2002.

\section{References}

[Be] J. Becker, Löwnersche Differentialgleichung und quasikonform fortsetzbare schlichte Funktionen, J. Reine Angew. Math. 255 (1972), 23-43.

[Br] A. A. Brodskiū, Quasiconformal extension of biholomorphic mappings, in: Theory of Mappings and Approximation of Functions, Naukova Dumka, Kiev, 1983, 3-34 (in Russian).

[Ch] M. Chuaqui, Applications of subordination chains to starlike mappings in $\mathbb{C}^{n}$, Pacific J. Math. 168 (1995), 33-48.

[Gr-Ha-Ko] I. Graham, H. Hamada and G. Kohr, Parametric representation of univalent mappings in several complex variables, Canad. J. Math. 54 (2002), 324-351.

[Gu] K. Gurganus, $\Phi$-like holomorphic functions in $\mathbb{C}^{n}$, Trans. Amer. Math. Soc. 205 (1975), 389-406.

[Ha] H. Hamada, Starlike mappings on bounded balanced domains with $C^{1}$-plurisubharmonic defining functions, Pacific J. Math. 194 (2000), 359-371.

[Ha-Ko1] H. Hamada and G. Kohr, Subordination chains and the growth theorem of spirallike mappings, Mathematica (Cluj) 42 (65) (2000), 153-161.

[Ha-Ko2] - - - , The growth theorem and quasiconformal extension of strongly spirallike mappings of type $\alpha$, Complex Variables 44 (2001), 281-297.

[Ha-Ko3] - - - Simple criterions for strongly starlikeness and quasiconformal extension, submitted.

[Ko] G. Kohr, Using the method of Löwner chains to introduce some subclasses of biholomorphic mappings in $\mathbb{C}^{n}$, Rev. Roumaine Math. Pures Appl. 46 (2001), 743-760.

[Pf1] J. A. Pfaltzgraff, Subordination chains and univalence of holomorphic mappings in $\mathbb{C}^{n}$, Math. Ann. 210 (1974), 55-68. 
[Pf2] J. A. Pfaltzgraff, Subordination chains and quasiconformal extension of holomorphic maps in $\mathbb{C}^{n}$, Ann. Acad. Sci. Fenn. Ser. A I Math. 1 (1975), 13-25.

[Pf-Su] J. A. Pfaltzgraff and T. J. Suffridge, Close-to-starlike holomorphic functions of several variables, Pacific J. Math. 57 (1975), 271-279.

[Pom] C. Pommerenke, Univalent Functions, Vandenhoeck \& Ruprecht, Göttingen, 1975 .

[Por1] T. Poreda, On the univalent holomorphic maps of the unit polydisc of $\mathbb{C}^{n}$ which have the parametric representation, I-the geometrical properties, Ann. Univ. Mariae Curie-Skłodowska Sect. A 41 (1987), 105-113.

[Por2] - On the univalent holomorphic maps of the unit polydisc of $\mathbb{C}^{n}$ which have the parametric representation, II-necessary and sufficient conditions, ibid., $114-121$.

[Por3] -, On the univalent subordination chains of holomorphic mappings in Banach spaces, Comment. Math. 128 (1989), 295-304.

[Re-Ma] F. Ren and J. Ma, Quasiconformal extension of biholomorphic mappings of several complex variables, J. Fudan Univ. Natur. Sci. 34 (1995), 545-556.

[Sa] S. Saks, Theory of the Integral, Warszawa, 1937.

[Su1] T. J. Suffridge, The principle of subordination applied to functions of several variables, Pacific J. Math. 33 (1970), 241-248.

[Su2] - Starlike and convex maps in Banach spaces, ibid. 46 (1973), 474-489.

[Su3] - Starlikeness, convexity and other geometric properties of holomorphic maps in higher dimensions, in: Lecture Notes in Math. 599, Springer, 1976, 146-159.

[Vä] J. Väisälä, Lectures in n-dimensional Quasiconformal Mappings, Lecture Notes in Math. 229, Springer, New York, 1971.

Faculty of Engineering

Kyushu Kyoritsu University

1-8 Jiyugaoka, Yahatanishi-ku

Kitakyusu 807-8585, Japan

E-mail: hamada@kyukyo-u.ac.jp
Faculty of Mathematics

Babeş-Bolyai University

1 M. Kogălniceanu St.

3400 Cluj-Napoca, Romania

E-mail: gkohr@math.ubbcluj.ro 